\title{
The role of gut microbiota in weight management by non-invasive interventions and bariatric surgery
}

\author{
Wojciech Marlicz ${ }^{1}$, Lucyna Ostrowska², Igor Łoniewski ${ }^{3}$ \\ ${ }^{1}$ Department of Gastroenterology, Pomeranian Medical University, Szczecin, Poland \\ ${ }^{2}$ Department of Dietetic and Clinical Nutrition, Medical University, Bialystok, Poland \\ ${ }^{3}$ International Pharmaceutical Consulting sp. z o.o. sp. k., Szczecin, Poland
}

\begin{abstract}
Obesity has become an emerging global issue responsible for an increased risk of cardiovascular diseases and cancer. Gut microbiota is one of biological factors involved in the pathogenesis of obesity and metabolic disorders. Microbiome alteration has been associated with chronic endotoxemia and microinflammation, insulin resistance and hyperinsulinemia - factors playing an important role in the development of metabolic disorders such as non-alcoholic steatohepatitis (NASH), diabetes mellitus (DM) and dyslipidemia. Bariatric interventions are an important part of obesity treatment. Surgical interventions are the most effective procedures. Endoscopy assisted techniques (intragastric balloon or duodenal-endoluminal sleeve) are also very promising, clinically tested procedures. Dietary interventions changing gut microbiota are more frequently used. However, our knowledge about the mechanisms of weight loss triggered by these interventions is incomplete. Recent experimental and clinical studies provide novel data that bariatric surgery modulates intestinal microbiome and changes its potential of energy harvest in the gut. Surgically altered microbiota in the short term exert profound but beneficial metabolic effects in humans. However, long-term consequences of such modulation are unknown. Recent data from randomized clinical trials support the notion that dietary interventions and supplementation of pre- and probiotics favorably modulate gut microbiota and augment weight loss after bariatric procedures.
\end{abstract}

Key words: gut microbiota, obesity, bariatric surgery, intragastric balloon, probiotics.

\section{Introduction}

In recent years significant progress has been achieved in our understanding of the pathogenesis of obesity and metabolic syndrome [1-3]. Environmental and genetic factors do not explain the increasing prevalence of metabolic disorders. With the development of molecular techniques to study gut microbiota, our understanding of microbiome has expanded enormously. Intestinal microbiota is a major player controlling metabolic balance in the body being responsible for efficient harvest, storage and expenditure of energy derived from food [4]. Experimental studies both in bacteria-free animal models as well as in humans confirm the importance of microbial factors in regulation of cell metabolism and proliferation $[5,6]$. Moreover, the manipulation of gut microbiota has been linked to weight modification both in animals and humans. The recent study has shown that bariatric intervention profoundly alters gut microbiota and influences host-microbial metabolic crosstalk [7]. It has been suggested that the beneficial metabolic effect of weight loss observed after surgical and endoscopic interventions resulted in microbiota modulation. Therefore, understanding the role of gut microbiota in the management of obesity seems to be of importance. Moreover, our better understanding of host-microbial crosstalk might lead to development of new strategies in the treatment of obesity. Finally, modulation of microbiota with pre- or probiotics before or after weight loss procedures might augment their long-term health effect. Therefore, targeting the microbiome in obesity should be viewed as an important factor with a potential to influence prognostic outcomes. Manipulation of microbiome offers new strategies to decrease the prevalence of metabolic disorders. 


\section{Obesity and metabolic syndrome: contemporary threats to human health}

Obesity and metabolic syndrome are currently viewed as the most important medical problems. These disorders are not only responsible for an increased risk of cardiovascular diseases and cancer but also for the risk of developing different gastrointestinal disorders. The most common ones include disorders of gallbladder and bile ducts, pancreas and colon, gastro-esophageal reflux disease (GERD), functional syndromes (e.g. functional dyspepsia and irritable bowel syndrome) [8] and liver diseases (e.g. non-alcoholic steatohepatitis [NASH]) [9]. Microbiome alterations in the gut caused by western type diet and sedentary lifestyle have been linked with an increased risk of cancer development. It has been described that patients diagnosed with cancer have a different microbial pattern in comparison to healthy individuals [10]. The metabolic profile of microbiota in cancer patients shifts towards production of genotoxic, mutagenic and carcinogenic compounds. Moreover, altered microbiota are capable of activating different food-derived pro-carcinogens in the gastrointestinal tract. Gut microbes shape the bile acid composition and promote de novo synthesis of carcinogens. Also the metabolic clearance of the liver could be affected by microbial components in the gut. Gut microbiota can also interfere with hormonal and nervous systems of an individual capable to affect the whole body homeostasis [11].

\section{The gut microbiota as an important metabolic organ in the human body}

The microbiota exert pleiotropic role in the human gastrointestinal tract. The fermentation of undigested food residuals and enterocyte-derived mucus are among the major digestive functions of gut microbiota. Microbiome, due to its functional complexity and residual mass in the gut, is frequently described as the "microbial organ". Microbiome contains 10 times more cells and 100 times more genes than the human organism and is involved in many important enzymatic and metabolic reactions. Its role in the development and regulation of immune and nervous systems is very important [12]. Detailed metabolic pathways regulated by microorganisms have just recently been discovered and described. As the majority of microbes exist in the gastrointestinal tract, they will not grow in culture media in vitro, thus molecular techniques are needed for their detailed identification and characterization. Based on molecular analysis it is known that anaerobic bacteria greatly outnumber aerobic ones with dominant species belonging to Bacteroides, Bifidobacterium, Eubacterium, Clostridium and Lactobacterium phyla. Aerobic bacteria, among them Enterococcus and Entero- bacteriaceae less abundantly inhabit the digestive tract. Laboratory observations reveal that the microbial pattern is unique in each individual and could be viewed as molecular fingerprint. The major role of microbiota is to harvest energy from food passing through the GI tract. This microbial process affects the regulation of total body mass and content of visceral fat. The microbial organ is capable of inducing immunological and inflammatory pathways resulting in uncontrolled epithelial cell proliferation and cancer. Microorganisms affect the tumor's metastatic potential and response to chemotherapy [13]. Mechanisms being described include modulation of tumor microenvironment, maintaining gut barrier integrity, macrophage priming and formation of new vessels.

Another interesting aspect of gut-microbe interaction is the regulation of gut peristalsis. As the components of gut-associated lymphoid tissue (GALT) are altered in germ-free animals, the number of enterocytes is also reduced. Microscopic observations reveal longer intestinal villas and shallow cryptal space in sterile, bacteria-free animals. As a consequence, the digestive function of bacteria-free animals is impaired and less efficient in comparison to conventionally colonized mice.

\section{Microbiota - intestinal barrier and metabolic endotoxemia in obesity}

Another important role of gut microbiota is the maintenance of the gut barrier structure and function [14]. Several factors may alter intestinal microbiota and cause dysbiosis in the gut. The most important factors disrupting the gut microbiota include high fat and protein diet and low fiber diet [15]. Standard "western" diet, rich in saturated fats and simple carbohydrates alter microbiome in a relatively short time. Commonly used medicines (e.g. antibiotics, non-steroidal anti-inflammatory drugs [NSAIDs] and proton pump inhibitors - PPIs) also transiently disturb the gut microbiota composition [16]. The repeated antibiotic therapy has been linked with excess body mass and obesity as well as cancer. Similarly, long-term PPIs use has been associated with weight gain. Other important factors affecting microbial composition in the gut are mental or physical stress and enteric infections. The role of microbiota in the pathogenesis of metabolic syndrome has been assessed in numerous experimental and clinical studies. The role of microbiota has been implicated in the regulation and maintenance of the gut barrier function and integrity. Transplantation of intestinal microbiota to bacteria-free mice is associated with obesity and insulin resistance development [17]. These observations confirm the important role of microbiome in harvesting the energy from food in the gastrointestinal tract. Moreover, bacteria-free mice transplanted with microbiota derived from obese mice gain weight faster than mice transplanted with 
"lean" type microbiota [4,5]. Gut microbiome in obese mice is more efficient in harvesting energy from the food. These animals possess more short chain fatty acids (SCFAs) in the digestive tract than lean animals. Genetically obese animals have a different microbial count than lean animals, characterized by Bacteroidetes decrease and Firmicutes increase [4]. However, the exact mechanism regulating the metabolic function of microbiota is far from being completely elucidated. Gut microbiota affects the utilization of energy by skeletal muscles as well as glucose metabolism in the liver. The development of fat tissue is also regulated by the microbial factors $[3,6]$.

Although the microbial profile differs according to the metabolic status of an individual it is still difficult or even impossible to measure its composition using routine diagnostic methods. However, its diversity could serve as an intermediate marker of the healthy microbial ecosystem. The hypothesis that the more diverse gut microbial population, the healthier is an individual has been confirmed by experimental and clinical observations. In contrast, dysbiosis has been linked with less diverse and pathogenic microbiota and is linked to obesity and metabolic disorders [3].

In overweight and obese humans, the ratio of Bacteroidetes to Firmicutes is changed [3]. The diet rich in protein and simple carbohydrates but deficient in fiber is associated with a decrease in Bifidobacterium species. In the gut of obese subjects, gram positive bacterial species e.g. Clostridia and Mollicutes dominate. Mollicutes possess an unique ability to harvest energy from food residues which is further stored in fat tissue. The ability of bacteria to translocate into the bloodstream and activate inflammatory cascades is also relevant. Mollicutes (especially Mycoplasma) have been found in various tissues and organs. Dysbiosis is associated with an increase in intestinal barrier permeability. The gut barrier consists of several proteins (zonula occludens-1 [ZO-1], desmosomes, occludin) forming tight and gap junctions. Dysbiosis in the gut promotes dysregulation of zonulin and occludin synthesis and loss of barrier integrity. As a consequence, bacterial antigens and bacteria derived lipopolysaccharides (endotoxins) leak through the gut to the bloodstream and mesenteric lymph nodes. Endotoxemia as well as SCFAs activate multiple processes in the human resulting in several metabolic complications [18]. Main metabolic pathways activated by gut microbiota were described previously [19].

All metabolic processes induced by microbiota directly affect total body mass. Paradoxically malnutrition can occur in obese subjects. The obese individual with malnutrition in order to fulfill bodily energy requirements has to deliver more energy with new portions of food to be digested. This is responsible for weight gain. Of course the genetic background of an individual is also important. The presence of inherited genes promoting energy extraction has been precisely described.

\section{Microbiota - insulin resistance and chronic microinflammation}

Special attention should be paid to the consequences of metabolic endotoxemia in peripheral tissues: a) generation of inflammatory milieu in visceral adipose tissue, and b) insulin resistance development. The low grade endotoxemia in subjects diagnosed with obesity and metabolic syndrome components has been well documented [18]. Intestinal Gram negative bacteria secrete lipopolysaccharides (LPS) - bacterial wall endotoxin capable of inducing systemic inflammation. This process is mediated by macrophages that efficiently phagocytose gut derived LPS and migrate to peripheral tissues causing inflammation. As a consequence, pathological release of inflammatory cytokines by adipocytes and macrophages follows decreased synthesis of adiponectin and increased release of leptin and resistin. Both these adipokines possess the capability of inducing inflammation and stimulating growth of cancerous cells [20]. Chronic microinflammation generate pro-acidic and pro-inflammatory environment. Insulin resistance is followed by increased production of insulin and insulin-like growth factors (IGF-1 and IGF-2). Insulin-like growth factors stimulate cell proliferation, induce apoptosis and affect the expression of proteins regulating the cell cycle. Increased secretion of IGFs and insulin is associated with higher prevalence of cancers of various organs, for example: i) prostate, ii) mammary gland, and iii) colon. Moreover, the prognosis of these cancers has been affected by the circulating insulin levels. Interestingly, Laron dwarfs with a mutation of insulin-like growth hormone in the liver are naturally protected against cancer and diabetes mellitus.

The simplified scheme presenting the prevalence of obesity and related metabolic disorders among the Polish population in regard to human microbiome is presented in Figure 1.

Below we discuss the important therapeutic options in the management of obesity.

\section{The role of gut microbiota manipulation in metabolic disease}

Experimental studies performed in animals with knock-outs of several innate immunity components revealed the important role of intestinal microbiota in the pathogenesis of obesity, liver steatosis and diabetes mellitus. Modulation of microbiota or innate immunity components in these animals has a protective effect of leads to complete regression of the disease [21].

Experimental studies performed in humans assessing the role of gut microbiota in the management of metabolic disturbances also deliver interesting results. Transplantation of stool microbiota from lean to obese subjects per- 
8-10 million people in Poland fullfil the criteria of metabolic syndrome.

In Poland $70 \%$ of adults have abnormal lipid profiles.

Several million Polish people suffer either from impared glucose tolerance or diabetes mellitus.

Every second person in Poland is either overweight or obese.

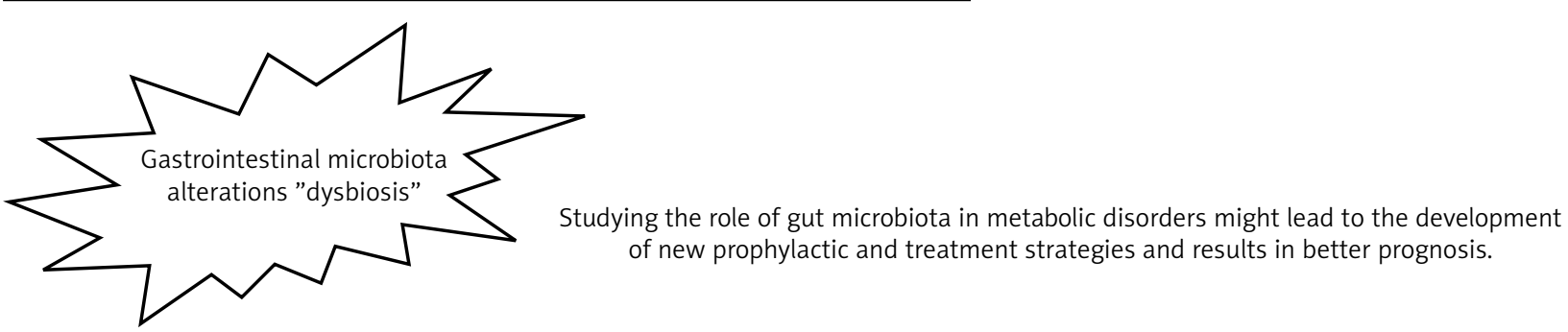

Figure 1. Obesity and metabolic syndrome are important medical problems in Poland and worldwide. Gastrointestinal microbiota (microbiome) is a key player in the pathogenesis of metabolic diseases $[2,11,17,19]$

formed in FATLOSE study resulted in decreased serum triglycerides levels and insulin resistance. However, this procedure failed to document weight loss effect in transplanted individuals during six weeks of the observational period. The strengths of this study included extensive molecular analysis of gut microbiota in transplanted patients [22]. FATLOSE 2 trial with a longer follow-up has already been initiated. The stool microbial transplantation serves as a good example of the beneficial potential of intestinal microbiota modulation. However, the safety of this procedure as well as costs and ethical acceptance have been questioned. Therefore, the application of probiotics and prebiotics in everyday clinical practice based on available literature as well as personal experience seems to be the best alternative. Commercially available probiotics with good safety profiles and documented clinical efficacy are the best examples. The detailed description of stool transplantation in the treatment of metabolic and autoimmune disease is beyond the scope of this paper and has been recently described by the authors elsewhere [23].

\section{The manipulation of gut microbiota by non-invasive interventions}

The dream to achieve significant weight loss with pharmacological agents remains an elusive goal only. Majority of drugs despite their efficacy in early clinical trials have already been withdrawn from the market due to numerous adverse events. New drugs (Lorcaserin and a combination of phentermine and topiramate) reduce hunger and await safety confirmation in clinical trials in the European population (both drugs have been already registered in the U.S.A.).

Neglecting the importance of gut microbiota could be an important factor limiting the efficacy of dietary regimens in weight management. The data obtained in ongoing Human Microbiome Project delivered novel evidence of the role of gut microbiota manipulation in the management of metabolic disorders. The potential modulators of gut microbiota include diet and prebiotics with probiotics.

\section{Diet}

Metabolic beneficial effects of energy restriction, physical activity and behavioral changes can be mediated by microbiota [24]. High protein and low carbohydrate diet reduces total fecal SCFAs in obese subjects. It is associated with reduction of bacteria involved in butyrate production (Eubacterium rectale, Roseburia spp., bifidobacteria) [25]. Santacruz et al. [26] found that energy restriction and exercise in overweight adolescents increased the number of Bacteroides fragilis and Lactobacillus and decreased the counts of Bifidobacterium longum and $B$. adolescentis. Interestingly, higher weight loss was connected with greater changes of gut microbiota. Nadal et al. [27] revealed several changes in fecal bacteria composition in overweight and obese adolescents involved in a multidisciplinary obesity treatment program. These changes were associated with the level of weight loss. The adolescents lost more weight and had also reduced total fecal energy loss.

The vegetarian diet improves several metabolic parameters (fasting and postprandial glycemia, weight reduc- 
tion, glucose and lipids metabolism) and also modulates gut microbiota (reduction of Firmicutes/Bacteroidetes ratio) [28]. It is important to stress that studies assessing the dietary impact combined with physical exercise on gut microbiota due to the methodological issues are not easy to design and perform. There is no single microbiological biomarker that can be used to assess the efficacy of weight reducing strategies. It has been suggested that the presence of mucin-degrading bacteria Akkermansia muciniphila in the human gut is associated with a better health and immune status [29]. In healthy individuals these bacteria compose approximately $3-5 \%$ of the whole microbiome [29] and their presence has been inversely correlated with body mass [30] and diabetes mellitus type 1 [31]. Everard et al. [32] found that administration of prebiotic oligofructose improved A. muciniphila number and metabolic profile in mice. Additionally, it was found that treatment with $A$. muciniphila reversed fat-mass gain induced by high-fat as well as metabolic endotoxemia, adipose tissue inflammation, and insulin resistance. The increase in intestinal levels of endocannabinoids that control inflammation, the gut barrier, and gut peptide secretion was also observed as the effect mediated by viable bacteria. On the other hand, Wills et al. [33] using next-generation sequencing to examine the fecal microbiota composition in patients with inflammatory bowel disease during an inactive disease phase and a subsequent exacerbation did not demonstrate general changes in gut microbiota composition or diversity in both groups of cohorts. They found a large increase in the relative number of Bacteroides fragilis or Akkermansia muciniphila during the active phase of disease in some patients. However, this important topic requires further studies.

The role of physical activity in addition to dietary weight loss programs is also important to investigate. Intensive physical training has been associated with reduced gastrointestinal blood flow, tissue hyperthermia and hypoxia leading to possible alterations of GI microbiota and gastrointestinal barrier function [34]. Clarke et al. [35] recently described the increased diversity of gut microbiota as a result of exercise associated with high protein diet in professional rugby players. These results indicate that the level of microbial biodiversity could be considered as a biomarker or indicator of health. Therefore, implementing probiotics into dietary regimes has been proposed as a novel and promising strategy. Lamprecht et al. [2] showed that 14 weeks' period of multi-species probiotics supplementation in trained men normalized stool zonulin concentrations (a marker of enhanced gut permeability) in comparison to a placebo group. Also the results of other studies with endurance athletes documented beneficial effects of dietary interventions on cytokines and immune-marker panel improvements and positive effects on redox biology and decrease in GI symptoms [34].

\section{Prebiotics}

Prebiotics (e.g. fructooligosaccharides and inulin) are non-digestible food ingredients that stimulate the growth and activity of bacteria in the gastrointestinal tract. Prebiotics serve as an energetic fuel to colonic epithelial cells and intestinal bacteria. Prebiotics also stimulate the secretion of SCFAs and release of anti-inflammatory cytokines in Peyer patches. Moreover, the synthesis of glucagon-like peptides (GLP-1 an GLP-2) is also increased by prebiotics. GLP proteins play an important role in the regulation of fat and carbohydrates metabolism. GLP-1 and -2 are insulin sensitizers in peripheral tissues diminishing the severity of insulin resistance. GLP-2 stimulates the synthesis of gut barrier structural proteins $\mathrm{ZO}-1$ and occludin. Another beneficial action of prebiotics is the induction of mucin secretion. The role of mucins in the GI tract is pleiotropic and has been described in detail elsewhere. Another important action of prebiotics is the regulation of satiety. Numerous studies assessing the efficacy of prebiotics in the treatment and prophylaxis of metabolic diseases have been already published. The clinical usefulness of arabinoxylan and inulin has been studied in the prevention and treatment of obesity, impaired glucose tolerance, type 2 diabetes mellitus, and non-alcoholic steatohepatitis [36]. Of special interest is inulin, a prebiotic naturally derived from plants and fructooligosaccharides. After ingestion, inulin has the natural potential to enhance the growth of Bifidobacteria in the GI tract. Inulin - in vivo - affects the secretion of GLP-1, ghrelin and peptide YY. Inulin has also been shown to be responsible for increasing the number of anti-inflammatory bacteria $F$. prausnitzii and decreasing the number of Bacteroides and Propionibacterium in healthy women. However, it is important to note that all of these studies have some limitations including a short observational period (up to 3-4 weeks) and relatively small study groups. More compelling evidence comes from longer intervention studies of prebiotics action. Administration of inulin-type fructan prebiotic for 12 months resulted in weight reduction and decrease in total fat mass in healthy young volunteers. Fructan administration to obese pre-menopausal women also resulted in weight reduction. Other studies deliver further evidence that prebiotics favor the weight loss and its maintenance [37]. As mentioned above, the mechanism of prebiotic action is complex but it is suggested that it is mediated by regulation of endocannabinoid system resulting in decreased gut barrier permeability, endotoxemia and fat storage. Since most of such evidence elucidating the mechanism of prebiotic action comes from animal studies, it is difficult to confirm full potential of prebiotic in the treatment of obesity in humans. However, their application in clinical practice in the management of obesity and related complications seems to be rational and very promising [38]. 


\section{Probiotics}

Probiotics are life bacteria, which have a beneficial effect on human health. Their supplementation is associated with more diverse and functional microbiota in the gut allowing for optimal harvest, storage and expenditure of energy from the food ingested. Probiotics mainly contain Gram-positive bacteria (e.g. Bifidobacterium spp., Lactobacillus spp., Lactococcus spp., Pediococcus spp.). Management of gut microbiota with probiotics influence body mass, but precise mechanisms have not been fully explained. One of the possible explanations of probiotic action is their aid in the maintenance of the intestinal barrier, inhibition of bacterial translocation from the gut and decrease of inflammatory reactions. As a consequence, the tissue utilization of insulin is enhanced and insulin resistance is decreased. The type of probiotic bacteria ingested seems to be very important as some bacterial species advertised as probiotics have been reported to increase body mass. This is supported by the results of meta-analysis showing that Lactobacillus acidophilus, fermentum and ingluviei administration was associated with weight gain in animals [39]. In contrast, consumption of L. plantarum and L. gasseri resulted in weight loss. The mechanism of probiotic action responsible for induction of weight loss may be explained by suppression of various hormonal pathways. Lam et al. [40] found that supplementation of animal diet with L. plantarum $299 \mathrm{v}$ resulted in $41 \%$ decrease of circulating leptin levels leading to enhanced cardioprotection [40]. The leptin lowering effect of $L$. plantarum $299 v$ has also been previously described in humans with elevated cholesterol levels [41]. Other studies described the beneficial effect of selected probiotic species (L. acidophilus NCFM and L. gasseri SBT2055) in the treatment of overweight individuals with visceral obesity and impaired glucose tolerance. The visceral and subcutaneous fat deposits as well as BMI were diminished in subjects treated with probiotics. It should be noted that most of commercially and clinically applicable antibiotics are associated with weight gain [39].

It has been suggested that subjects diagnosed with obesity with an increased risk of cancer development might benefit from prophylactic probiotic supplementation. Numerous experimental studies have shown the benefit from probiotic administration in the prevention of cancers. Ishikawa et al. [42], based on observation of 398 males and females diagnosed with two tumors in the colon and undergoing surgery, reported that administration of $L$. casei for 2-4 years prevents the relapse of atypical neoplasia in the colon [42]. In another study, authors reported: i) 12 weeks' observation of 43 patients after endoscopic polypectomy and 37 patients diagnosed with cancer consuming Lactobacillus rhamnosus GG, Bifidobacterium animalis ssp. lactis DSM15954 and inulin with oligofructose - an intervention positively modulated intestinal flora with improvement of the barrier function and decrease in pro-inflammatory cytokines, ii) 38 healthy male volunteers supplemented for four weeks with Lactobacillus rhamnosus LC705 and Propionibacterium freudenreichii ssp. shermanii JS - a significant increase in the count of Lactobacillus and Propionibacterium species in the stool as well as a decrease in the activity of $\beta$-glucosidase ( $\beta$-glucosidase has been shown to be responsible for promotion of cancer growth and progression). In addition, a recently published study analyzing the risk of developing cancer among 477122 men and women revealed a possible protective role of dairy products on the colorectal cancer risk [43]. Moreover, consumption of multispecies probiotics containing Bifidobacteria has been linked to a decrease in insulin resistance and improvement of liver steatosis and atherosclerosis [44].

The effect of noninvasive and invasive interventions capable of modulating human microbiome and their effect on body mass is presented in Figure 2.

Below we discuss weight loss management by means of invasive interventions.

\section{Changes of gut microbiota by invasive weight loss interventions}

\section{Endoluminal endoscopic therapy}

Endoscopic procedures are minimally invasive options for the treatment of obesity. Endoscopic bariatric procedures are safe, reversible, cost and clinically effective. The modern endoscopic techniques can be divided into either restrictive or malabsorptive (e.g. gastrointestinal bypass procedure). One of the most common endoscopic procedure is the intragastric balloon resulting in short-term weight loss. Modern fluid filled silicone gastric balloons are inserted with the aid of endoscope and durable for the maximum time of 6 months. The aim of this treatment is to initiate the sense of satiety secondary to stomach dilatation. The short-term weight loss effect was observed in both retrospective as well as prospective clinical trials. In addition, improvements in blood pressure, glycemic control and lipid levels as secondary outcomes as well as reduction in prevalence of co-morbidities was shown. Although short-term results were very optimistic, the long-term benefit of this procedure has been questioned. The majority of patients (> 75\%) without dietary or physical activity treatment plan gained weight after two years of balloon removal. Thus, this technique should be used to reduce weight in morbidly obese subjects before surgical intervention [45]. Close collaboration between the patient and the dietitian should be recommended. A common adverse effect of balloon placement is the exacerbation of GERD. It is suggested that that 

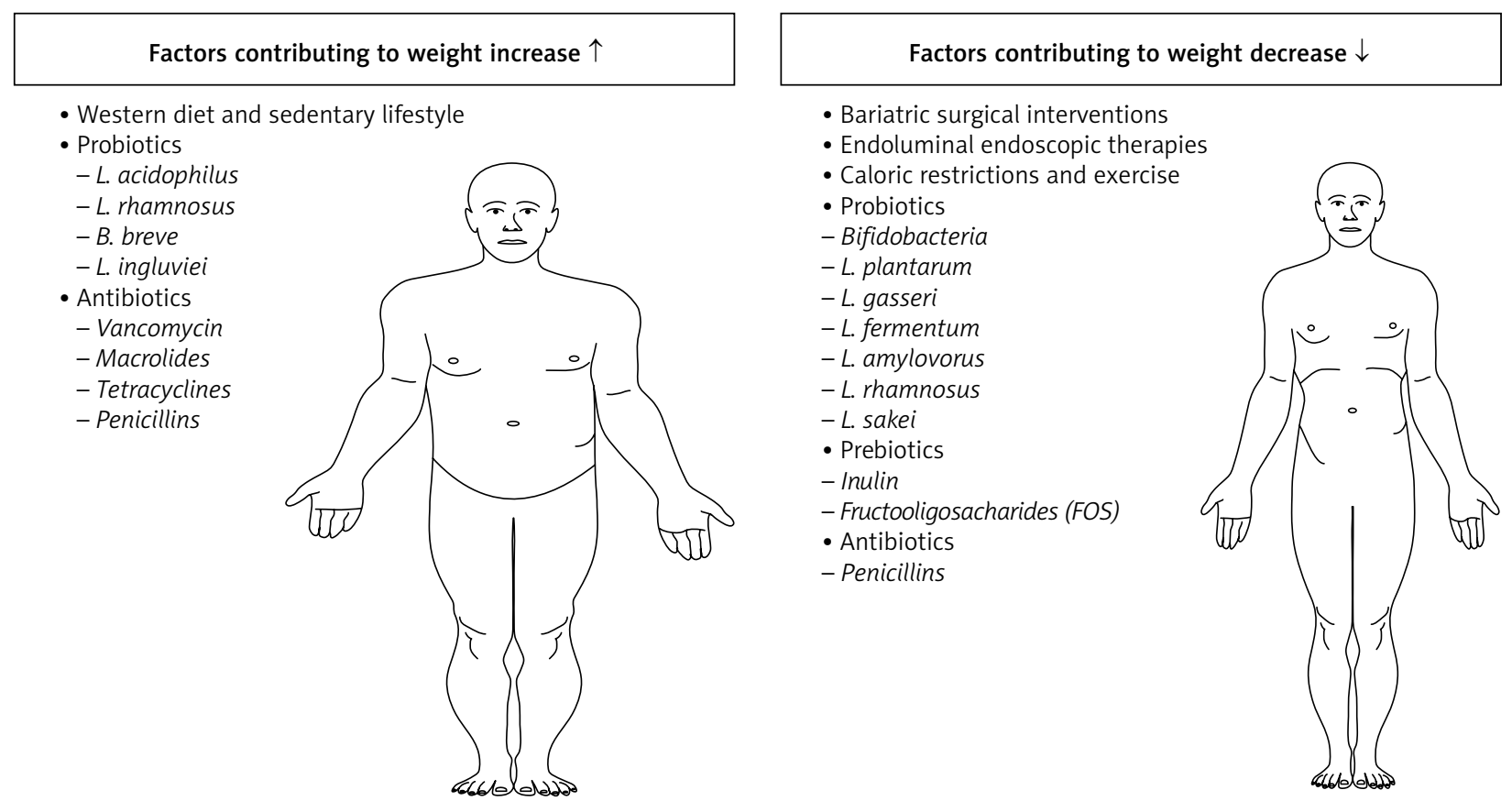

Figure 2. Factors contributing to weight increase and loss. All of the above factors have the capabilities to modulate gut microbiota contributing to weight gain or loss [7,35,39,48-50]

balloon placement is associated with small intestine bacterial overgrowth (SIBO). However, this hypothesis requires confirmation. Another endoscopic technique performed in obesity treatment is transoral gastroplasty (TOGA System). This system utilizes an endoscopic stapling device in order to form a special pouch in the course of lesser curvature of the stomach. However, this technique is not used in our country and its future worldwide is uncertain. The effect of intragastric balloon placement as well as TOGA system on gut microbiota or small intestinal bacterial overgrowth has not been studied.

Of great clinical interest is the application of EndoBarrier sleeve endoscopic gastrointestinal bypass device. Despite some initial technical obstacles, its therapeutic usefulness in the treatment of diabetes mellitus and obesity has been confirmed in the pilot studies. Thus, duodenal nutrient exclusion by means of EndoBarrier placement is a promising therapeutic approach to diabetes and obesity treatment [46]. The effects of this technique on gut microbiota are unknown.

\section{Bariatric surgery}

The surgical procedures in obesity treatment could be classified as restrictive, malabsorptive or restrictive/malabsorptive. The outcome assessment of bariatric surgery should be focused not only on the weight loss effect but also on metabolic benefits. Therefore, more than 30 years ago, Buchwald $\mathrm{H}$. proposed the following definition of metabolic surgery and/or intervention: "metabolic surgery is the operative manipulation of a normal organ or organ system to achieve a biological result for a potential health gain" [47]. Recent questionnaires sent out to almost forty International Federations for the Surgery and Obesity and Metabolic Disorders revealed more than 340 thousand bariatric surgeries recorded in 2008 worldwide with over $90 \%$ of them performed laparoscopically. The most commonly performed surgical procedures were laparoscopic adjustable gastric banding (AGB; 42\%), laparoscopic standard Roux-Y gastric bypass (RYGB; 39\%) and total sleeve gastrectomies (4\%). These procedures are very effective in the management of obesity and result in approximately $40 \%$ weight loss and improvement or even regression of metabolic disease in the majority of treated patients [47]. However, the precise mechanism responsible for weight loss is poorly understood. Relevant factors include ameliorated eating habits, changed pattern of nutrient absorption, changed motility and gastric emptying, modified bile acid composition and hormonal secretion. Recent studies have shown the role of microbiota in weight loss after bariatric surgery. Zhang et al. [48] revealed the decrease in the number of Firmicutes after RYGB compared with controls. In addition, a significant increase in the number of Proteobacteria after RYGB was observed.

Li et al. [7] found that the RYGB procedure resulted in reduction of weight and food intake in laboratory animals and significantly affected the changes of gut microbiota. The microbiome analysis revealed a significant increase in Proteobacteria and a decrease in Firmicutes and Bacteroidetes. These changes are associated with the following processes: i) increasing intestinal fermentation of oligo- 
saccharides, ii) production of p-cresol, iii) activation of certain amines capable of mechanistically initiate weight loss. These authors also identified Gammaproteobacteria abundantly colonizing gut immediately after surgery. Gammaproteobacteria were very efficient "metabolizers" of carbohydrates. This observation is important as food in RYGB-treated individuals skips the digestive phase in duodenum and goes directly into the jejunum. The associations between the type of surgery and microbial composition in the GI tract was found also in other studies. Furet et al. [49] reported that the number of Escherichia coli in the gut correlated negatively with the body mass index and the presence of F. prausnitzii was associated with the lack of inflammatory markers after RYGB. It has also been reported that RYGB was associated with a decreased number of Firmicutes and increased numbers of Gammaproteobacteria. Although, observed changes and shifts in the microbiome might seem positive in the short-term, the long-term effect of this phenomenon is not known. Li et al. [7] suggested that increased numbers of Gammaproteobacteria in a longer time might favor apoptosis of epithelial cells and exert a negative effect on health. This hypothesis was confirmed by the results of a study which found fecal cytotoxicity after the RYGB procedure. On the other hand, epidemiological studies confirm beneficial effects of bariatric surgery on the decreased risk of cardiovascular disease and cancer development. Although experimental studies clearly demonstrated that bariatric procedures profoundly changed the intestinal microbiome, the exact role of this phenomenon requires further in-depth analysis.

In light of the above-described findings, very interesting are the studies assessing the role of probiotics in the

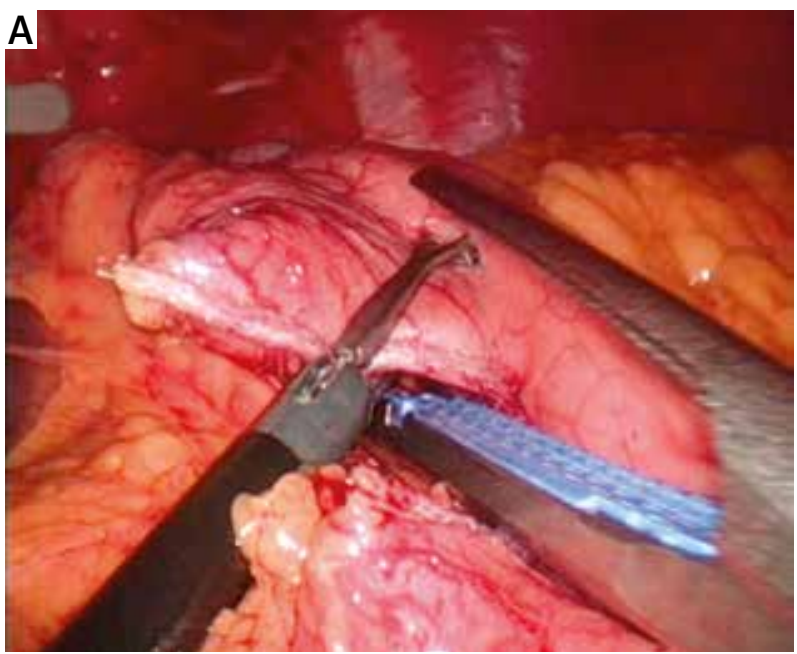

bariatric perioperative period. The positive and protective effect of probiotics administered before or after surgical intervention on reduction of the risk of infection is well known. However, the results of studies evaluating the use of probiotics to improve outcomes of bariatric surgical interventions are less evident. It was reported that Lactobacillus species administration was associated with better vitamin $B_{12}$ availability and a greater weight loss effect after RYGB. In addition, the lower bacterial overgrowth in intestine was observed [50].

This study also confirmed the observations that obese patients have an overabundance of the hydrogen-producing bacterial species in their gastrointestinal tract [50]. It has been suggested that hydrogen transfer between bacterial species might be an important mechanism regulating energy uptake in obese subjects. Therefore, monitoring the gut microbiota and intestinal bacterial overgrowth could be of benefit in assessing the weight change potential in obese subjects after bariatric procedures. The photographic examples of bariatric surgical intervention are presented in Figure 3.

\section{Conclusions}

The microbiome is a key player in the pathogenesis of obesity and metabolic disorders. Altered microbiota evoke multiple reactions of the immune system and induces inflammatory reactions in the colon, jejunum, liver and fat tissue. As a consequence, insulin resistance in peripheral tissues develops. The number of people diagnosed with obesity and metabolic disorders has increased significantly in recent years. Most common diseases associated with obesity include type 2 diabetes mellitus and non-alcoholic

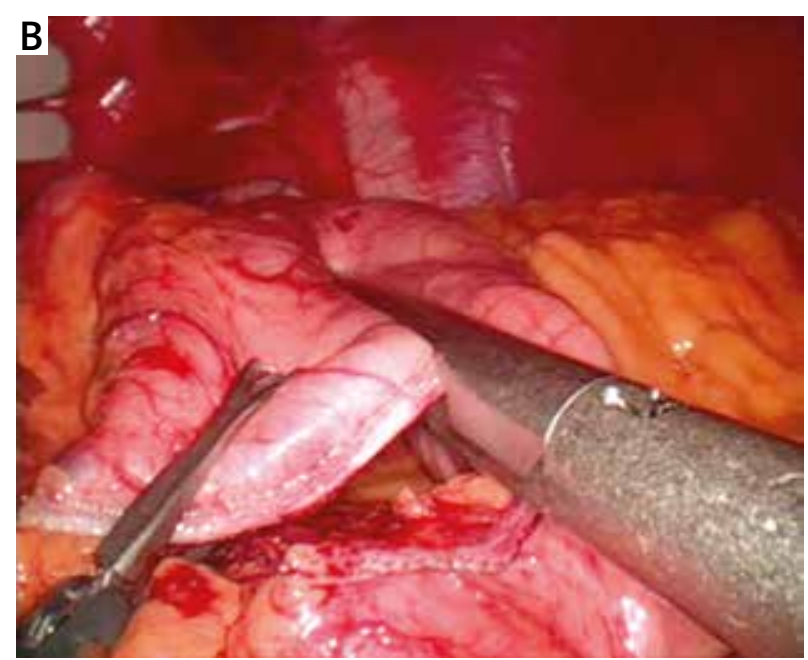

Figure 3. Obesity and metabolic syndrome are important medical problems worldwide. Supplementing obese patients with lactobacillus probiotics results in greater weight loss and less small intestinal bacterial overgrowth after RYGB bariatric intervention (ref. [50]). Photo: Roux-en-Y Gastric Bypas (RYGB) laparoscopic bariatric surgery (Author: dr Krzysztof Kaseja, Department of General and Vascular Surgery, Specialist Hospital, Szczecin-Zdunowo; photo - courtesy of dr Krzysztof Kaseja) 
steatohepatitis. Obese individuals have also a significantly increased risk of developing cancer and cardiovascular diseases. The precise mechanism responsible for weight loss after bariatric interventions is not completely clarified. It has been suggested that their effect at least in part is mediated by modulation of intestinal microbiota. The observed displacement in the microbiome in the short term after surgery is positive, however the long-term effect of bariatric interventions is not known. Changes of gut microbiota after prebiotics and probiotics administration may have a beneficial effect on health of obese subjects undergoing bariatric surgery. Although more studies on this topic are needed, we believe that doctors should already implement this knowledge into everyday clinical practice. Physicians and nurses should also take active part in educating patients and lay public to consider the benefits of microbiome modulation in obesity and metabolic syndrome. The perspective is healthier life with a lower risk of cardiovascular disease and cancer.

\section{Acknowledgments}

We are grateful to dr Krzysztof Kaseja at the Department of General and Vascular Surgery, Specialist Hospital, Szczecin-Zdunowo for sharing experience and the photographic documentation taken during Roux-en-Y Gastric Bypass bariatric laparoscopic surgery (performing surgeon: dr Krzysztof Kaseja).

\section{Disclosure}

Authors report no conflict of interest.

\section{References}

1. Fukuda $\mathrm{S}$, Ohno H. Gut microbiome and metabolic diseases. Semin Immunopathol 2014; 36: 103-114.

2. Nicholson JK, Holmes E, Kinross J, et al. Host-gut microbiota metabolic interactions. Science 2012; 336: 1262-1267.

3. Ley RE. Obesity and the human microbiome. Curr Opin Gastroenterol 2010; 26: 5-11.

4. Turnbaugh PJ, Ley RE, Mahowald MA, Magrini V, Mardis ER, Gordon JI. An obesity-associated gut microbiome with increased capacity for energy harvest. Nature 2006; 444: 1027-1031.

5. Ley RR, Bäckhed F, Turnbaugh P, Lozupone CA, Knight RD, Gordon JI. Obesity alters gut microbial ecology. Proc Natl Acad Sci U S A 2005; 102: 11070-11075.

6. Karlsson CL, Onnerfalt J, Xu J, Molin G, Ahrné S, Thorngren-Jerneck $K$. The microbiota of the gut in preschool children with normal and excessive body weight. Obesity (Silver Spring) 2012; 20: 2257-2261.

7. Li Jia V, Ashrafian H, Bueter M, et al. Metabolic surgery profoundly influences gut microbial-host metabolic cross-talk. Gut 2011; 60: 1214-1223.

8. Nguyen DM, El-Serag HB. The epidemiology of obesity. Gastroenterol Clin North Am 2010; 39: 1-7.

9. Tilg H, Diehl AM. NAFLD and extrahepatic cancers: have a look at the colon. Gut 2011; 60: 745-746.
10. Marchesi JR, Dutilh BE, Hall N, et al. Towards the human colorectal cancer microbiome. PLoS 2011; 6: e20447.

11. Stachowicz N, Kiersztan A. The role of gut microbiota in the pathogenesis of obesity and diabetes. Postepy Hig Med Dosw (online) 2013; 67: 288-303.

12. Hooper LV, Littman DR, Macpherson AJ. Interactions between the microbiota and the immune system. Science 2012;336: 1268-1273.

13. Viaud S, Saccheri S, Mignot G, et al. The intestinal microbiota modulates the anticancer immune effects of cyclophosphamide. Science 2013; 342: 971-976.

14. Quigley EM. Small intestinal bacterial overgrowth: what it is and what it is not. Curr Opin Gastroenterol 2014; 30: 141-146.

15. Marlicz W, Zawada I, Starzyńska T. Irritable bowel syndrome - irritable bowel or irritable mind? Pol Merkur Lekarski 2012; 32: 64-69.

16. Wallace JL, Syer S, Denou E, et al. Proton pump inhibitors exacerbate NSAID-induced small intestinal injury by inducing dysbiosis. Gastroenterology 2011; 141: 1314-1322.

17. Cani P, Delzenne NM. Gut microflora as a target for energy and metabolic homeostasis. Curr Opin Clin Nutr Metab Care 2007; 10: 729-734.

18. Mazmanian SK, Kasper DL. The love-hate relationship between bacterial polysaccharides and the host immune system. Nat Rev Immunol 2006; 6: 849-858.

19. Marlicz W, Ostrowska L, Łoniewski I. Flora bakteryjna jelit i jej potencjalny związek z otyłością. Endokrynol Otyl Zab Przem Mat 2013; 1: $20-28$ [in Polish].

20. Tilg $H$, Kaser A. Gut microbiome, obesity and metabolic syndrome. J Clin Invest 2011; 121: 2126-2132.

21. Vijay-Kumar M, Aitken JD, Carvalho FA. Metabolic syndrome and altered gut microbiota in mice lacking Toll-like receptor 5 . Science 2010; 328: 228-231.

22. Vrieze A, Van Nood E, Holleman F, et al. Transfer of intestinal microbiota from lean donors increases insulin sensitivity in individuals with metabolic syndrome. Gastroenterology 2012; 143: 913-916.

23. Ostrowska L, Marlicz W, Łoniewski I. Transplantacja mikroflory jelitowej w leczeniu otyłości i zaburzeń metabolicznych - metoda nadal ryzykowna i niepotwierdzona wynikami badań klinicznych. Forum Zab Metabol 2013; 4: 1-7 [in Polish].

24. Erejuwa OO, Sulaiman SA, Ab Wahab MS. Modulation of gut microbiota in the management of metabolic disorders: the prospects and challenges. Int J Mol Sci 2014; 15: 4158-4188.

25. Duncan SH, Belenguer A, Holtrop G, Johnstone AM, Flint HJ, Lobley GE. Reduced dietary intake of carbohydrates by obese subjects results in decreased concentrations of butyrate and butyrate-producing bacteria in feces. Appl Environ Microbiol 2007; 73: 1073-1078

26. Santacruz A, Marcos A, Warnberg J, et al. Interplay between weight loss and gut microbiota composition in overweight adolescents. Obesity (Silver Spring) 2009; 17: 1906-1915.

27. Nadal I, Santacruz A, Marcos A, et al. Shifts in clostridia, bacteroides and immunoglobulin-coating fecal bacteria associated with weight loss in obese adolescents. Int J Obes (Lond) 2009; 33: 758-767.

28. Kim MS, Hwang SS, Park EJ, Bae JW. Strict vegetarian diet improves the risk factors associated with metabolic diseases by modulating gut microbiota and reducing intestinal inflammation. Environ Microbiol Rep 2013; 5: 765-775.

29. Derrien M, Vaughan EE, Plugge CM, de Vos WM. Akkermansia muciniphila gen.nov., sp. nov., a human intestinal mucin-degrading bacterium. Int J Syst Evol Microbiol 2004; 54: 1469-1476.

30. Karlsson CL, Onnerfält J, Xu J, Molin G, Ahrné S, Thorngren-Jerneck K. The microbiota of the gut in preschool children with normal and excessive body weight. Obesity (Silver Spring) 2012; 20: 2257-2261. 
31. Everard A, Lazarevic V, Derrien M, et al. Responses of gut microbiota and glucose and lipid metabolism to prebiotics in genetic obese and diet-induced leptin-resistant mice. Diabetes 2011; 60: 2775-2786.

32. Everard A, Belzer C, Geurts L, et al. Cross-talk between Akkermansia muciniphila and intestinal epithelium controls diet-induced obesity. Proc Natl Acad Sci USA 2013; 110: 9066-9071.

33. Wills ES, Jonkers DMAE, Savelkoul PH, Masclee AA, Pierik MJ, Penders J. Fecal microbial composition of ulcerative colitis and Crohn's disease patients in remission and subsequent exacerbation. PLoS One 2014; 9: e90981.

34. Lamprecht M, Frauwallner A. Exercise, intestinal barrier dysfunction and probiotic supplementation. Med Sport Sci 2012; 59: 47-56.

35. Clarke SF, Murphy EF, O'Sullivan O, et al. Exercise and associated dietary extremes impact on gut microbial diversity. Gut 2014; pii: gutjnl-2013-306541; doi: 10.1136/gutjnl-2013-306541.

36. Delzenne NM, Neyrinck AM, Bäckhed F, Cani PD. Targeting gut microbiota in obesity: effects of prebiotics and probiotics. Nat Rev Endocrinol 2011; 7: 639-646.

37. Delzenne NM, Neyrinck AM, Cani PD. Gut microbiota and metabolic disorders: how prebiotic can work? Br J Nutr 2013; 109: S81-S85.

38. Diamant M, Blaak EE, de Vos WM. Do nutrient-gut-microbiota interactions play a role in human obesity, insulin resistance and type 2 diabetes? Obes Rev 2011; 12: 272-281.

39. Angelakis E, Merhej V, Raoult D. Related actions of probiotics and antibiotics on gut microbiota and weight modification. Lancet Infect Dis 2013; 13: 889-899.

40. Lam V, Su J, Koprowski S, et al. Intestinal microbiota determine severity of myocardial infarction in rats. FASEB J 2012; 26: 1727 1735.

41. Naruszewicz M, Johansson ML, Zapolska-Downar D, Bukowska H. Effect of Lactobacillus plantarum 299v on cardiovascular disease risk factors in smokers. Am J Clin Nutr 2002; 76: 1249-1255.

42. Ishikawa H, Akedo I, Otani T, et al. Randomized trial of dietary fiber and Lactobacillus casei administration for prevention of colorectal tumors. Int J Cancer 2005; 116: 762-767.

43. Murphy N, Norat T, Ferrari P, et al. Consumption of dairy products and colorectal cancer in the European Prospective Investigation into Cancer and Nutrition (EPIC). PLoS One 2013; 8: e72715.

44. Bischoff SC (ed.). Probiotika, Präbiotika und Synbiotika. Georg Thieme Verlag KG, Stuttgart, New York 2009, pp. 247-248.

45. Miras AD, le Roux CW. Can medical therapy mimic the clinical efficacy or physiological effects of bariatric surgery? Internat J Obes (Lond) 2014; 38: 325-333.

46. Habegger KM, Al-Massadi O, Heppner KM, et al. Duodenal nutrient exclusion improves metabolic syndrome and stimulates villus hyperplasia. Gut 2014; 63: 1268-1246.

47. Buchwald H, Oien DM. Metabolic/bariatric surgery worldwide 2008. Obes Surg 2009; 19: 1605-1611.

48. Zhang H, DiBaise JK, Zuccolo A, et al. Human gut microbiota in obesity and after gastric bypass. Proc Natl Acad Sci U S A 2009; 106: 2365-2370.

49. Furet JP, Kong LC, Tap J, et al. Differential adaptation of human gut microbiota to bariatric surgery-induced weight loss: links with metabolic and low-grade inflammation markers. Diabetes 2010; 59: 3049-3057.

50. Woodard GA, Encarnacion B, Downey JR, et al. Probiotics improve outcomes after Roux-en-Y gastric bypass surgery: a prospective randomized trial. J Gastrointest Surg 2009; 13: 1198-1204. 